\title{
Characteristic time scales of mixing, mass transfer and biomass growth in a Taylor vortex algal photobioreactor
}

\author{
Xi Gao, Bo Kong, R. Dennis Vigil* \\ Department of Chemical \&5 Biological Engineering, Iowa State University, Ames, IA \\ 50011
}

\begin{abstract}
Recently it has been demonstrated that algal biomass yield can be enhanced using fluid flow patterns known as Taylor vortices. It has been suggested that these growth rate improvements can be attributed to improved light delivery as a result of rapid transport of microorganisms between light and dark regions of the reactor. However, Taylor vortices also strongly impact fluid mixing and interphase (gas-liquid) mass transport, and these in turn may also explain improvements in biomass productivity. To identify the growth-limiting factor in a Taylor vortex algal photobioreactor, experiments were performed to determine characteristic time scales for mixing and mass transfer. By comparing these results with the characteristic time scale for biomass growth, it is shown that algal growth rate in Taylor vortex reactors is not limited by fluid mixing or interphase mass transfer, and therefore the observed biomass productivity improvements are likely attributable to improved light utilization efficiency. Keywords: mass transfer, Taylor vortex reactor, Taylor-Couette flow,
\end{abstract}

\footnotetext{
${ }^{*}$ Corresponding author

Email address: vigil@iastate.edu (R. Dennis Vigil)
} 
photobioreactors

\section{Introduction}

Development of more efficient algal photobioreactors (PBRs) is driven by increasing interest in algaculture for the production of fuels, chemicals, food, animal feed, and medicine (Spolaore et al., 2006). Even for high-volume applications that require pond systems, such as the production of biofuels, PBRs play an important auxiliary role in maintaining pond cultures. Moreover, because of the better control of mixing and containment of growth media and feed gas offered by PBR systems, the prospect exists for using industrial $\mathrm{CO}_{2}$ waste streams for cultivation of microalgae (Morweiser et al., 2010). Consequently, there is significant incentive to design and operate algal PBRs with high biomass productivity and conversion efficiency.

Many factors affect performance of PBRs, such as the type of PBR, culture media, temperature, $\mathrm{pH}$, microorganism used, $\mathrm{CO}_{2}$ mass transfer, $\mathrm{O}_{2}$ accumulation, mixing, light intensity and light/dark cycles (Kumar and Das, 2012). Among these, the major limiting factors for growth of microalgae are usually light availability and interphase mass transfer. Light limitations caused by absorption and scattering can occur even in dilute cell cultures, depending upon the distance of the light path through the reactor and the intensity of the incident radiation ( $\mathrm{Hu}$ and Richmond, 1996). Near the reactor irradiated surface, algal radiative exposure is usually adequate or in excess, whereas a dark volume with insufficient light for photosynthesis to occur often resides only a few centimeters or less from the 
irradiated surface, depending on the cell concentration (Kong and Vigil, 2014). For this reason, a key factor in the design of PBRs is the incorporation of mechanisms to periodically transport cells between light and dark regions of the reactor (mixing-induced light/dark cycles) in order to efficiently utilize radiation near the illuminated reactor surface and distribute it evenly to microorganisms (Hu and Richmond, 1996; Ugwu et al., 2005; Sobczuk et al., 2006; Huang et al., 2014).

Mixing induced light/dark (L/D) cycles usually occur at frequencies on the order of $1 \mathrm{~Hz}$ or less, which is significantly lower than the minimum frequencies required to produce the flashing light effect $(>25 \mathrm{~Hz})$. Nevertheless, it has been demonstrated that photosynthesis can be enhanced by low frequency L/D cycles (Miller et al., 1964; Grobbelaar et al., 1994; Morweiser et al., 2010; Huang et al., 2014; Takache et al., 2015). In flat-plate (Hu and Richmond, 1996; Janssen et al., 2003), bubble column (Merchuk et al., 1998), and airlift bioreactors (Merchuk et al., 1998; Degen et al., 2001), fluid transport between light and dark regions of the reactor occurs primarily via diffusive processes resulting from bubble-induced mixing, and consequently these reactors do not generate the characteristic L/D cycles required to more evenly distribute light to microorganisms (Liao et al., 2014). As a result, some conventional PBR designs incorporate foils or baffles to generate coherent L/D cycles (Degen et al., 2001; Ugwu et al., 2005; Liao et al., 2014).

As an alternative approach for generating L/D cycles for culturing algae, Miller et al. (1964) used a Taylor-Couette device (fluid confined to the annulus between two concentric cylinders, with the inner cylinder 
rotating) to generate toroidal Taylor vortices that rapidly and reliably shuttle fluid between the poorly lit inner cylinder and the well-illuminated outer cylinder. Although they performed only short time scale experiments, they were able to demonstrate that the rate of photosynthesis (via oxygen measurements) increased with increasing cylinder rotation speed, and they attributed these enhancments to increases in the L/D frequencies experienced by microorganisms.

More recently, Taylor vortex PBRs have been used to culture Chlorella vulgaris to very high concentrations in both batch (Kong et al., 2013) and continuous cultures (Kong and Vigil, 2013), and it has been demonstrated that the biomass yields are significantly greater than those obtained in the absence of Taylor vortices (i.e. an annular bubble column with no cylinder rotation). However, it should be noted that gas-liquid mass transport is also significantly enhanced by the presence of Taylor vortices (Ramezani et al., 2015), and as a result the question arises as to whether algal growth rate enhancements in these devices is attributable primarily to the creation of L/D cycles, to improved interphase mass transport of carbon dioxide and oxygen, or to a combination of both effects. Hence, the purpose of this report is to describe the findings of a series of batch culture experiments designed to determine characteristic times for liquid mixing, gas-liquid mass transfer, and algal biomass growth in a Taylor-Couette PBR for a range of reactor inner cylinder rotation speeds, feed gas flow rates, and feed gas compositions. As a result of these experiments, it can be concluded that improved light delivery is apparently the only plausible physical mechanism that can explain the enhanced algal 
growth rates observed in Taylor vortex PBRs.

\section{Materials and Methods}

\subsection{Organism, Media, and Subculture}

All experiments were carried out using Chlorella vulgaris (UTEX \#265) grown in a modified M8-a medium having the composition described by Kliphuis et al. (2010). Subcultures were maintained in 250-mL shake flasks at room temperature with an incident photon flux of 10-20

$\mu \mathrm{mol} \cdot \mathrm{m}^{-2} \cdot \mathrm{s}^{-1}$ provided by compact fluorescent light bulbs, and agitated by a gas mixture of air enriched with 5\% (molar) carbon dioxide. The culture medium was sterilized with a $0.22 \mu \mathrm{m}$ sterile filter after the $\mathrm{pH}$ was adjusted to a value of $6.7 \pm 0.1$ by adding $2 \mathrm{~N}$ potassium hydroxide solution. In the Taylor vortex PBR, the solution $\mathrm{pH}$ depends upon the carbon dioxide gas-liquid equilibrium, and it was consistently measured during culture conditions to be in the range of 7.0-8.0. In all experiments reported here, the PBR was inoculated at cell dry biomass concentration of 0.067 $\mathrm{g} / \mathrm{L}$, as determined from optical density measurements.

\subsection{Photobioreactor}

A 3D rendering of the Taylor-Couette PBR used in this study can be found in Gao et al. (2015), and a schematic diagram of the batch culture system is shown in Figure 1. The fixed outer cylindrical reactor wall and surrounded heating jacket were constructed using transparent Plexiglas. The diameters of the rotating inner and fixed outer cylinders were 7.62 and $10.16 \mathrm{~cm}$, respectively, resulting in an annular gap width of $1.27 \mathrm{~cm}$. The 
length of the reactor $(48 \mathrm{~cm})$ provided an annular working volume of approximately 1.7 L. The stainless steel inner cylinder was rotated by a stepper motor (Applied Motion Products, STM24SF) mounted at the top of the reactor. Sterilization of the annular reaction chamber was achieved by filling it with a solution of $70 \%$ alcohol followed by rinsing with autoclaved deionized water. The reactor temperature was maintained at 37 ${ }^{\circ} \mathrm{C}$ by circulating water through the reactor jacket using a NESLAB Instruments EX-221 water bath circulator. The reactor was enclosed along its length by four Hydrofarm eco-4441B flat light panels, each of which was comprised of four T5 fluorescent light tubes. The photosynthetic photon flux on the surface of the reactor (external surface of the heating jacket) was measured to be $450 \mu \mathrm{mol} \cdot \mathrm{m}^{-2} \cdot \mathrm{s}^{-1}$ using a LICOR LI-190 2 quantum sensor connected to a LI-250 light meter. The feed gas, consisting of a binary mixture of nitrogen and carbon dioxide (6-12 mol \% $\left.\mathrm{CO}_{2}\right)$ at a total flow rate of $85-510 \mathrm{ml} / \mathrm{min}(0.05-0.30 \mathrm{vvm})$ was first passed through a sterile filter and then pumped into the reactor via four $40-\mu \mathrm{m}$ sureseal miniature mufflers (McMaster-Carr, type $\mathrm{H}$ ) arranged symmetrically inside the bottom end cap of the reactor. The inlet gas flow rate of each gas component was controlled by digital gas flow controllers and the off-gas flow rate was measured using a gas flow meter. Further details concerning the apparatus and procedure can be found in Kong et al. (2013).

\subsection{Experimental Measurements}

The mole fractions of carbon dioxide and oxygen in the off-gas were continuously measured and recorded throughout each experiment at 10 minute intervals by passing the off-gas through a gas analyzer (Quantek 
Instrumetns, 902P, Grafton, MA) connected downstream of a desiccant purifier (Drierite, L68GP). The biomass concentration was monitored at 6-12 hour intervals by extracting culture samples from the reactor using a syringe and measuring optical density. In addition, dry biomass was determined from $10 \mathrm{~mL}$ reactor broth samples that were centrifuged at $3400 \mathrm{rpm}$ for 12 minutes, freeze-dried for 48 hours, and weighed using a balance with $0.1 \mathrm{mg}$ resolution. The relationship between optical density $\left(\mathrm{OD}_{680}\right)$ and dry biomass $\left(C_{b}\right)$, as previously determined from hundreds of measurements in our earlier work (Kong et al., 2013; Kong and Vigil, 2013), is well represented by the following correlation,

$$
C_{b}(\mathrm{~g} / \mathrm{L})=0.33\left(\mathrm{OD}_{680}\right), \quad \mathrm{R}^{2}=0.997
$$

Because of the reliability of the above relation, dry biomass was not weighed at every sampling interval; in some instances biomass concentration was computed from optical density measurements, thereby reducing the culture volume loss during the course of an experiment.

Elemental composition (C, H, N and S) of dry biomass samples was determined using an elemental analyzer (Perkin Elmer 2100 Series II CHN/S Analyzer). Dry biomass samples were first freeze-dried for 48 hours and subsequently heated in an oven at $65{ }^{\circ} \mathrm{C}$ for an additional 48 hours to completely remove water before they were analyzed for composition. Three measurements were performed for each sample in order to improve accuracy. The mean elemental composition of the biomass produced under various experimental conditions (cylinder rotation speed, gas feed rate and composition) was: $45.92 \% \mathrm{C}, 7.46 \% \mathrm{H}, 5.59 \% \mathrm{~N}$ and $0.67 \% \mathrm{~S}$. 


\section{Calculation of Characteristic Time Scales}

\subsection{Rate Controlling Step}

Transport of gas phase species such as oxygen and carbon dioxide to and from microorganisms in general involves several mechanistic steps including transit across a gas boundary layer to the gas-liquid interface, dissolution at the gas-liquid boundary, transit across a liquid boundary layer near the interface, convective liquid mixing, transit across a biofilm at the microorganism surface, and consumption/production in the microorganism itself (Kraakman et al., 2011). Commonly, the resistances in the gas film and in bulk liquid mixing are neglected, as these quantities are usually small compared to the resistances in liquid films at interface boundaries. In principle, the mass transfer coefficients for the gas-liquid interface $\left(k_{L}\right)$ and the biofilm $\left(k_{b}\right)$ both depend upon fluid flow in the reactor, but computational fluid dynamics calculations of the Kolmogorov length for typical conditions in a Taylor vortex reactor reveal that most algal microorganisms with characteristic sizes on the order of $10 \mu \mathrm{m}$ or less are too small to be sensitive to fluid shear, and as a result it is unlikely that $k_{b}$ is impacted by the inner cylinder rotation speed (Thomas and Gibson, 1990). Therefore, it can be anticipated that changes in cylinder rotation speed affect interphase mass transport primarily through modulation of $k_{L}$.

In batch culture systems, which are inherently unsteady, the rate-limiting step may change with time. In such cases, it is useful to compare characteristic time scales for each mechanistic step in order to identify the rate-limiting process. In this work we identify these time scales

for liquid phase mixing $\left(t_{\text {mix }}\right)$ of dissolved $\mathrm{CO}_{2}$, transport of $\mathrm{CO}_{2}$ across the 
liquid film at the gas-liquid interface $\left(t_{M T}\right)$, and consumption of $\mathrm{CO}_{2}$ by microorganisms, as reflected by the characteristic time for biomass growth $\left(t_{g}\right)$.

\subsection{Characteristic Mixing Time}

Fluid mixing behavior is characterized by a mixing time, which is defined as the time required to achieve a specific degree of homogeneity after a trace pulse has been injected into the reactor (Gavrilescu and Roman, 1996). In this work, the acid tracer method (Chisti, 1989; Kumar and Das, 2012) was used to measure the mixing time. The annulus was first filled with room temperature deionized water. Subsequently nitrogen was pumped through sparge stones into the reactor for 10 minutes to purge dissolved carbon dioxide. Thereafter, $5 \mathrm{~mL}$ of $2 \mathrm{M}$ HCL was added to the water to lower the $\mathrm{pH}$ to approximately 3.50 at steady state. The tracer (10 $\mathrm{mL}$ of $1 \mathrm{M} \mathrm{KOH}$ ) was then rapidly injected into the bottom of the reactor using a syringe, and the temporal $\mathrm{pH}$ response inside the annulus was recorded using a $\mathrm{pH}$ probe located at the top of the reactor. The time required for the $\mathrm{pH}$ to attain a value within $5 \%$ of the final steady state value $\left(t_{t r}\right)$ was then determined from $\mathrm{pH}$ time series measurements.

The protocal described above provides a good representation of the time required for the reactor to achieve spatial homogeneity from an initial condition with maximal fluid segregation (tracer located as far as possible from the detector). However, as we are primarily interested in the transport of $\mathrm{CO}_{2}$, this species enters the liquid growth medium not at a single source point far from the reactor exit but at many locations in the reactor via rapidly rising gas bubbles. Furthermore, since it is well-known that fluid 
mixing within Taylor vortices is much more rapid than is the mixing between vortices (Campero and Vigil, 1997), it is more appropriate to estimate the fluid mixing time by normalizing the $\mathrm{pH}$ response measurements described above by the number of Taylor vortices present in the system. The latter quantity can be estimated by making use of the analytical result that the axial wavelength $\lambda$ of a pair of vortices at the onset of Taylor vortex flow is given by $\lambda=2\left(r_{o}-r_{i}\right)$, where $r_{o}$ and $r_{i}$ are the radii of the inner and outer cylinders, respectively (Coles, 1965). Hence, the characteristic mixing time can be estimated as

$$
t_{\text {mix }}=\frac{t_{t r}\left(r_{o}-r_{i}\right)}{L}
$$

where $L$ is the fluid height in the reactor.

\subsection{Characteristic Gas-Liquid Mass Transfer Time}

Neglecting gas film resistance, the instantaneous volumetric rate of gas-liquid mass transfer $R_{M T}$ is given by

$$
R_{M T}=k_{L} a\left(C_{\mathrm{CO}_{2}}^{*}-C_{\mathrm{CO}_{2}}\right)
$$

where $C_{\mathrm{CO}_{2}}^{*}$ and $C_{\mathrm{CO}_{2}}$ are the dissolved $\mathrm{CO}_{2}$ concentrations in the liquid at the gas-liquid interface and in the liquid bulk, respectively. The characteristic mass transfer time, $t_{M T}$, is therefore given by

$$
t_{M T}=\frac{1}{k_{L} a}
$$

where $k_{L}$ is the liquid side mass transfer coefficient and $a$ is the specific interfacial area. 
For an air-water system, the volumetric mass transfer coefficient for oxygen, $k_{L} a\left(\mathrm{O}_{2}\right)$, in a vertically-oriented Taylor vortex PBR at room temperature can be computed from correlations recently developed by Ramezani et al. (2015), given by:

$$
\begin{gathered}
\mathrm{Sh}=\frac{k_{L} a d_{b}^{2}}{D_{L}}=1.45 \times 10^{-7} \operatorname{Re}_{a}^{0.83}\left(\operatorname{Re}_{\theta}+1.47 \times 10^{4}\right)^{1.61}, \\
\frac{d_{b}}{r_{o}-r_{i}}=1.0 \times 10^{-5} \operatorname{Re}_{a}^{0.11}\left(\operatorname{Re}_{\theta}+3.3 \times 10^{4}\right)^{0.91} .
\end{gathered}
$$

In the above equations Sh is the Sherwood number, $d_{b}$ is the Sauter mean bubble diameter, $D_{L}$ is the diffusivity of the solute in the liquid phase, and $\operatorname{Re}_{a}$ and $\operatorname{Re}_{\theta}$ are the axial and azimuthal Reynolds numbers defined as

$$
\operatorname{Re}_{a}=\frac{2 u_{G}\left(r_{o}-r_{i}\right)}{\nu_{L}}
$$

and

$$
\operatorname{Re}_{a}=\frac{\omega r_{i}\left(r_{o}-r_{i}\right)}{\nu_{L}} .
$$

The axial Reynolds number $\operatorname{Re}_{a}$ is based upon the hydraulic diameter and superficial gas velocity, $u_{G}$, and the azimuthal Reynolds number $\operatorname{Re}_{\theta}$ is based on the inner cylinder speed and annular gap width. Because the gas holdup in vertical gas-liquid Taylor vortex reactors is low $(<\approx 3 \%)$, the fluid kinematic viscosity is used in both definitions (Gao et al., 2015). Experimental details concerning the development of the correlations given by Eqs. 5 and 6 can be found in Ramezani et al. (2015), who used a minimally invasive oxygen sensing system (PreSens Fibox 4 transmitter and Pst3 sensor spots) to measure time-dependent dissolved oxygen concentrations in response to step input changes. 
From Eqs. 4 - 8 it is possible to compute $t_{M T}$ based upon the mass transfer coefficient for oxygen. The characteristic mass transfer time based upon the mass transfer coefficient for carbon dioxide can also be computed by making use of the Higbie theory (Higbie, 1935) to determine the relationship between $k_{L} a\left(\mathrm{O}_{2}\right)$ and $k_{L} a\left(\mathrm{CO}_{2}\right)$ :

$$
k_{L} a\left(\mathrm{CO}_{2}\right)=k_{L} a\left(\mathrm{O}_{2}\right) \sqrt{\frac{D_{\mathrm{CO}_{2}}}{D_{\mathrm{O}_{2}}}},
$$

where $D_{\mathrm{CO}_{2}}$ and $D_{\mathrm{O}_{2}}$ are the diffusivities of carbon dioxide and oxygen in water. Because the correlations presented in Eqs. 5 and 6 are based upon experiments carried out at room temperature, whereas the culture experiments described here were performed at $37^{\circ} \mathrm{C}$, it is necessary to make use of the following relationships to estimate the temperature dependence of the diffusion coefficients (Tamimi et al., 1994; Han and Bartels, 1996):

$$
\log _{10} D_{\mathrm{CO}_{2}}=-2.047-\frac{541.2}{T}-\left(\frac{275.7}{T}\right)^{2},
$$

and

$$
\log _{10} D_{\mathrm{O}_{2}}=-4.410+\frac{773.8}{T}-\left(\frac{506.4}{T}\right)^{2} .
$$

Hence, the characteristic $\mathrm{CO}_{2}$ mass transfer time in the experiments discussed here can be expressed as

$$
t_{M T}=\frac{1}{k_{L} a\left(\mathrm{O}_{2}, 298 \mathrm{~K}\right)} \sqrt{\frac{D_{\mathrm{O}_{2}}(298 \mathrm{~K})}{D_{\mathrm{CO}_{2}}(310 \mathrm{~K})}},
$$

where $k_{L} a\left(\mathrm{O}_{2}, 298 \mathrm{~K}\right)$ can be determined from Eq. 5 .

\subsection{Characteristic Biomass Growth Time}

The characteristic biomass growth time $t_{g}$ can be estimated by assuming that the growth rate has first order dependence on the dissolved 
$\mathrm{CO}_{2}$ concentration, which is available at its equilibrium concentration $C_{\mathrm{CO}_{2}}^{*}$ (i.e. no transport limitations), leading to

$$
t_{g}=\frac{C_{\mathrm{CO}_{2}}^{*}}{Q_{\mathrm{CO}_{2}}} .
$$

In the above equation, $Q_{\mathrm{CO}_{2}}$ is the volumetric uptake rate of $\mathrm{CO}_{2}(\mathrm{~g} / \mathrm{L} \cdot \mathrm{s})$ by the microorganisms, which can be determined by analyzing the composition and flow rate of the off gas and comparing it with the feed gas. Alternatively, the $\mathrm{CO}_{2}$ uptake rate can be determined from the evolution of the dry biomass concentration provided that the biomass yield coefficient, $Y_{\mathrm{CO}_{2}}$ (biomass produced/ $\mathrm{CO}_{2}$ mass consumed), is known:

$$
Q_{\mathrm{CO}_{2}}=\frac{1}{Y_{\mathrm{CO}_{2}}} \frac{d C_{b}}{d t} .
$$

The yield coefficient in the experiments carried out here is estimated to have the value $Y_{\mathrm{CO}_{2}}=0.594$ based upon the elemental analysis of the dry biomass product described earlier. Hence, the characteristic biomass growth time can be expressed as (Doran, 1993):

$$
t_{g}=\frac{Y_{\mathrm{CO}_{2}} C_{\mathrm{CO}_{2}}^{*}}{d C_{b} / d t}=\frac{Y_{\mathrm{CO}_{2}} C_{\mathrm{CO}_{2}}^{*}}{\mu C_{b}}
$$

where $\mu$ is the specific growth rate defined as

$$
\mu=\frac{1}{C_{b}} \frac{d C_{b}}{d t}
$$

The evolution of the biomass concentration using Chlorella vulgaris in a batch-operated Taylor vortex PBR can be well represented by least-square fits of the logistic equation (Kong et al., 2013):

$$
C_{b}=\frac{C_{b, \infty}}{1+e^{-k\left(t-t_{o}\right)}}
$$


where $C_{b, \infty}$ is the asymptotic value of $C_{b}$ at long times, $k$ is a constant representing the maximal instantaneous growth rate (slope of the inflection point on the sigmoidal growth curve), and $t_{o}$ is the time at which the maximal instantaneous growth rate occurs. Combining Eqs. 15, 16, and 17 leads to the following expression:

$$
t_{g}=\frac{Y_{\mathrm{CO}_{2}} C_{\mathrm{CO}_{2}}^{*}\left(1+e^{-k\left(t-t_{o}\right)}\right)^{2}}{k C_{b, \infty} e^{-k\left(t-t_{o}\right)}} .
$$

In order to compute $t_{g}$ using either Eq. 13 or 18 , the $\mathrm{CO}_{2}$ equilibrium concentration, $C_{\mathrm{CO}_{2}}^{*}$, must be known. This quantity can be estimated from Henry's law,

$$
C_{\mathrm{CO}_{2}}^{*}=\frac{y_{\mathrm{CO}_{2}} P M_{\mathrm{CO}_{2}}}{H_{\mathrm{CO}_{2}}},
$$

where, $y_{\mathrm{CO}_{2}}$ is the mole fraction of carbon dioxide in the feed gas, $P$ is the total gas pressure, $M_{\mathrm{CO}_{2}}$ is the molecular weight of $\mathrm{CO}_{2}$, and $H_{\mathrm{CO}_{2}}$ is the Henry coefficient for carbon dioxide. The dependence of the latter quantity on temperature for water is given by Sander (2015):

$$
H_{\mathrm{CO}_{2}}=29.41 e^{-2400\left(\frac{1}{T}-\frac{1}{298}\right)} .
$$

\section{Results and Discussion}

\subsection{Mixing Time}

Figure 2 shows plots of the experimentally measured mixing time for different rotational speeds and gas flow rates. It is evident from these plots that the liquid mixing time, which ranged between approximately 10 - 30 seconds for the conditions shown in Fig. 2, decreases with increasing cylinder rotation speed as well as with increasing feed gas flow rate. These 
results are consistent with those reported by Racina et al. (2010), who measured mixing time for single phase Taylor-Couette flow in a reactor with similar geometry as the device used in this work $\left(r_{i}=3.79 \mathrm{~cm}\right.$, $r_{o}=5.0 \mathrm{~cm}$, and $L=39.0 \mathrm{~cm}$ ) using laser-optical methods.

\subsection{Interphase Mass Transfer Time}

As was discussed previously, an empirical correlation (Eqs. 5 - 8) for the oxygen volumetric mass transfer coefficient $k_{L} a\left(\mathrm{O}_{2}\right)$ in a vertical Taylor vortex reactor identical to the reactor used here was recently developed by Ramezani et al. (2015). By making use of this correlation and Eq. 9, plots for $k_{L} a\left(\mathrm{CO}_{2}\right)$ were generated as functions of the cylinder rotation speed and gas flow rate for the algal culture conditions investigated here, as shown in Figure 3. It is clear from these plots that $k_{L} a\left(\mathrm{CO}_{2}\right)$ increases linearly with gas flow rate, whereas it has a more complicated dependence upon cylinder rotation speed. Below $300 \mathrm{rpm} k_{L} a\left(\mathrm{CO}_{2}\right)$ is relatively insensitive to rotation speed, whereas it increases approximately linearly at higher rotation speeds. This phenomenon can be attributed to a shift in the relative importance of the contributions of gas-liquid slip velocity and turbulence dissipation rate to interphase mass transfer above $300 \mathrm{rpm}$, as is explained by Gao et al. (2015). As was discussed earlier, the characteristic time for $\mathrm{CO}_{2}$ gas-liquid mass transfer is simply the reciprocal of $k_{L} a$, and therefore based upon the plots in Fig. $3, t_{M T} \approx 1$ to 8 minutes, which is significantly greater than the values found for $t_{m i x}$.

Interphase gas-liquid mass transfer is also important with respect to oxygen, which is toxic to algal microorganisms if the $\mathrm{O}_{2}$ dissolved concentration becomes too large. With respect to $C$. vulgaris in particular, 
Märkl and Mather (1985) reported that saturation of the culture media using pure oxygen results in a dissolved oxygen concentration of 44.16 $\mathrm{mg} / \mathrm{L}$ at $20^{\circ} \mathrm{C}$ (475\% of the value for the concentration observed in air-saturated water), which in turn reduces the photosynthetic rate by $35 \%$ in an open pond system. The level of oxygen accumulation in the liquid culture media depends on the hydrodynamic conditions and PBR design. It has previously been reported that commonly used bubble column and airlift PBRs operate at a maximum dissolved oxygen concentration of approximately $115 \%$ of the value for water saturated with air, whereas dissolved oxygen concentrations reach as high as $400 \%$ of the value for water-air saturation in horizontal tubular PBRs (Mirón et al., 2002). In contrast, Figure 4 shows that even using a low gas flow rate, the maximum observed dissolved oxygen concentration, which occurs during periods of maximal photosynthetic activity, was only $90 \%$ of the water-air saturation value. As a result, it is reasonable to conclude that oxygen transport limitations do not inhibit algal growth in the Taylor vortex reactor experiments considered in this study.

\subsection{Biomass Growth Experiments}

Although algal growth rate in a Taylor vortex PBR depends upon many factors other than fluid mixing and interphase mass transfer, such as choice of culture medium and temperature, here we limit our considerations to the effects of the two most important operational parameters for these devices, namely the inner cylinder rotation speed and the feed gas flow rate (Gao et al., 2015). Both quantities strongly impact fluid flow patterns which in turn determine microorganism light exposure history (L/D cycles). 
In addition, a series of experiments was also carried out using constant gas volumetric flow rate but with different feed gas compositions. Biomass growth curves (and corresponding yield plots) showing the effects of cylinder rotation speed, gas flow rate, and gas composition are presented in Figure 5 .

In all experiments the biomass undergoes rapid initial growth followed by an approximately linear growth phase before leveling off at a maximum concentration of approximately 10-14 g/L at long times. These growth curves are typical of batch systems that must eventually become limited by supply of nutrients, and they can be well-approximated by the logistic equation 17 (best fits are shown as solid lines in Fig. 5). The instantaneous biomass yield can be computed by differentiating these best fit equations for biomass growth, i.e. yield $=d C_{b} / d t$, and these results are plotted as functions of the resident biomass concentration (instantaneous yields are shown in units of days rather than hours by assuming 24 hours of illumination/day) in Fig. 5 (b), (d), and (f).

The plots in Fig. 5 (a) and (b) demonstrate that the growth rate is insensitive to the feed composition when the $\mathrm{CO}_{2}$ concentration is greater than $6 \%$. As the feed concentration of $\mathrm{CO}_{2}$ increases, the dissolved concentration of $\mathrm{CO}_{2}$ should also increase according to Henry's law, provided that other factors remain unchanged. Because the total gas volumetric flow rate and cylinder rotation speed were fixed in the experiments depicted in Fig. 5 (a), it is reasonable to assume that the fluid flow patterns are identical in all the experiments, and hence the lack of sensitivity of the growth curves to gas feed composition for $\mathrm{CO}_{2}$ 
concentrations greater than $6 \%$ suggests that the growth rate is not limited by $\mathrm{CO}_{2}$ supply or interphase mass transport resistance for feed compositions with $\mathrm{CO}_{2}$ concentrations greater than approximately $8 \%$. In view of the fact that the mixing time $t_{m i x}$ was found to be significantly shorter than the interphase mass tranfer time $t_{M T}$, any limitation in the biomass growth rate can only be attributable to limitations in light availability, nutrients other than $\mathrm{CO}_{2}$, or some other factor. Further evidence that growth is not limited by $\mathrm{CO}_{2}$ supply for cases studied with feed $\mathrm{CO}_{2}$ concentrations greater than $8 \%$ is provided by temporal data for the $\mathrm{CO}_{2}$ uptake rate, which can be computed from measurements of the off-gas composition. Specifically, in the experiment carried out using a $\mathrm{CO}_{2}$ feed composition of $6 \%$ nearly all of the fed $\mathrm{CO}_{2}$ was consumed during an the interval of time corresponding to the maximum biomass growth rate. In contrast, the maximum $\mathrm{CO}_{2}$ uptake in cases using higher $\mathrm{CO}_{2}$ feed concentrations ranges between approximately $60 \%$ and $90 \%$.

Figures 5 (c) and (d) illustrate the effect of cylinder rotation speed for constant gas flow rate $(0.05 \mathrm{vvm})$ and composition $\left(10 \% \mathrm{CO}_{2}\right)$. Based upon Eq. 5, it is evident that increases in inner cylinder rotation speed lead to increases in the gas-liquid mass transfer coefficient. Furthermore, because the feed concentration of $\mathrm{CO}_{2}$ used in these experiments was much greater than $6 \%$, algal growth was not likely to be limited by $\mathrm{CO}_{2}$ supply or mass transfer resistance for any of the rotation speeds studied, and therefore the growth rate dependence on cylinder rotation speed can only be attributed to some other factor that is sensitive to hydrodynamics. Having eliminated mixing and mass transfer as causes, the remaining explanation for these 
observations is that increases in inner cylinder speed produce higher frequency L/D cycles due to faster rotation of the toroidal vortices around a circular axis passing through their cores, and these higher frequencies improve light utilization efficiency (Kong et al., 2013).

The effect of feed gas volumetric flow rate on biomass growth rate was also considered, and the results are shown in Figs. 5 (e) and (f) for constant cylinder rotation speed $(400 \mathrm{rpm})$ and feed gas composition $\left(10 \% \mathrm{CO}_{2}\right)$. Biomass growth rate is improved by increasing the feed gas flow rate from $0.05 \mathrm{vvm}$ to $0.2 \mathrm{vvm}$, whereas further increases in the flow rate do not appear to have any impact on biomass growth. As has already been discussed, biomass growth rate in experiments using sufficiently high $\mathrm{CO}_{2}$ concentrations in the feed gas is not limited by interphase mass transport, and hence the impact of increasing the gas flow rate must be explained in terms of fluid flow patterns and light delivery. In our previous work (Gao et al., 2015), it was shown that centrifugal force concentrates gas bubbles near the inner cylinder. The accumulation of bubbles near the inner cylinder slightly reduces the radial light path into the liquid growth medium by forcing the liquid to reside closer to the outer cylinder. It may also be possible that increases in gas flow rate (and consequently gas volume fraction near the inner cylinder) result in greater reflection of light off the inner cylinder wall and back into the liquid media. However, at higher gas flow rates Taylor vortices become significantly elongated (Gao et al., 2015), and this may have an adverse effect on L/D cycle frequency, which may explain why further increases in gas flow rate do not result in enhanced biomass growth rates. 


\subsection{Comparison of Characteristic Time Scales}

Evidence presented in the previous section suggests that neither fluid mixing nor gas-liquid mass transfer inhibit biomass growth in the Taylor vortex PBR. This view is further supported by computing the characteristic growth time using either Eq. 13 or Eq. 18 and comparing these with $t_{m i x}$ and $t_{M T}$ over an extended time during a culture experiment. As was discussed previously, the computation of $t_{g}$ assumes that dissolved $\mathrm{CO}_{2}$ is at its maximal (saturation) value of $C_{\mathrm{CO}_{2}}^{*}$. As a result, the experimentally determined values for $t_{g}$ depend primarily upon light delivery or nutrition limitations other than $\mathrm{CO}_{2}$.

Figure 6 (a) and (b) depict the evolution of the three characteristic time scales discussed above, for two experimental conditions (although these results are typical for all experiments performed with sufficient $\mathrm{CO}_{2}$ supply in the feed gas). For the duration of both experiments, the characteristic growth time is one to two orders of magnitude greater than the mass transfer and mixing time scales. Hence, neither mass transfer of $\mathrm{CO}_{2}$ from gas bubbles to the liquid growth medium nor liquid mixing limit biomass growth in the Taylor vortex PBR. Nevertheless, Fig. 5 (c) demonstrates that algal growth rates can be enhanced by increasing the reactor inner cylinder speed. Since mass transport effects have been excluded as a cause for these growth rate enhancements, the remaining probable explanation is that increased cylinder rotation speeds lead to better light delivery to microorganisms through an increase in L/D cycle frequency. 


\section{Conclusion}

An analysis and comparison of time scales computed for three key mechanistic steps in the production of algal biomass, including gas-liquid mass transfer, liquid mixing, and algal growth, supports the conclusion that fluid flow patterns in these systems accelerate growth through improved light delivery to microorganisms rather than by improved delivery of carbon dioxide. The analysis presented here provides a guide for deconvoluting the effects of mixing, mass transfer, and light delivery for other PBR designs as well, and such information could be useful for optimizing PBR design and operation.

\section{Acknowledgment}

Financial support was provided for this work by National Science Foundation grant CBET-1236676. 


\section{Bibliography}

1. Campero, R.J., Vigil, R.D. 1997. Axial dispersion during low Reynolds number TaylorCouette flow: intra-vortex mixing effects. Chem. Eng. Sci. 52, 3303--3310.

2. Chisti, M.Y. 1989. Airlift Bioreactors. Elsevier Applied Science, London.

3. Coles, D. 1965. Transition in circular Couette flow. J. Fluid Mech. 21, 385--425.

4. Degen, J., Uebele, A., Retze, A., Schmid-Staiger, U., Trosch, W. 2001. A novel airlift photobioreactor with baffles for improved light utilization through the flashing light effect. J. Biotechnol. 92, 89-94.

5. Doran, P.M., 1993. Design of reactors for plant cells and organs. Springer-Verlag, Berlin.

6. Gao, X., Kong, B., Vigil, R.D. 2015. CFD investigation of bubble effects on Taylor-Couette flow patterns in the weakly turbulent vortex regime. Chem. Eng. J. 270, 508-518.

7. Gavrilescu, M., Roman, R.V. 1996. Mixing times in an external-loop airlift bioreactor with static mixers. Acta Biotechnol. 16, 145-153.

8. Grobbelaar, J.U. 1994. Turbulence in mass algal cultures and the role of light-dark fluctuations. J. Applied Phyc. 6, 331-335.

9. Han, P., Bartels, D.M. 1996. Temperature dependence of oxygen diffusion in $\mathrm{H}_{2} \mathrm{O}$ and $\mathrm{D}_{2} \mathrm{O}$. J. Phys. Chem. 100, 5597-5602.

10. Higbie, R. 1935. The rate of absorption of a pure gas into still liquid during short periods of exposure. Trans. Am. Inst. Chem. Eng. 31, 365.

11. Hu, Q., Richmond, A. 1996. Productivity and photosynthetic efficiency of Spirulina platensis as affected by light intensity, algal density and rate of mixing in a flat plate photobioreactor. J. Appl. Phyc. 8, 139-145.

12. Huang, J., Li, Y., Wan, M., Yan, Y., Feng, F., Qu, X., Wang, J., Shen, G., Li, W., Fan, J., Wang, W. 2014. Novel flat-plate photobioreactors for microalgae cultivation with special mixers to promote mixing along the light gradient. Biores. Tech. 159, 8-16.

13. Janssen, M., Tramper, J., Mur, L.R., Wijffels, R.H. 2003. Enclosed outdoor photobioreactors: Light regime, photosynthetic efficiency, scale-up, and future prospects. Biotech. Bioeng. 81, 193-210.

14. Kliphuis, A.M.J., de Winter, L., Vejrazka, C., Martens, D.E., Janssen, M., Wijffels, R. 2010. Photosynthetic efficiency of Chlorella sorokiniana in a turbulently mixed short light-path photobioreactor. Biotech. Prog. 26, 687-696. 
15. Kong, B., Shanks, J.V., Vigil, R.D. 2013. Enhanced algal growth rate in a Taylor vortex reactor. Biotech. Bioeng. 110, 2140-2149.

16. Kong, B., Vigil, R.D. 2013. Light-limited continuous culture of Chlorella vulgaris in a Taylor vortex reactor. Environ. Prog. I\& Sus. Ener. 32, 884-890.

17. Kong, B., Vigil, R.D. 2014. Simulation of photosynthetically active radiation distribution in algal photobioreactors using a multidimensional spectral radiation model. Biores. Tech. 158, 141-148.

18. Kraakman, N.J.R., Rocha-Rios, J., van Loosdrecht, M.C.M. 2011. Review of mass transfer aspects for biological gas treatment. Appl. Microbiol. Biotech. 91, 873-886.

19. Kumar, K., Das, D. 2012. Growth characteristics of Chlorella sorokiniana in airlift and bubble column photobioreactors. Biores. Tech. 116, 307-313.

20. Liao, Q., Li, L., Chen, R., Zhu, X. 2014. A novel photobioreactor generating the light/dark cycle to improve microalgae cultivation. Biores. Tech. 161, 186-191.

21. Markl, H., Mather, M. 1985. Mixing and aeration of shallow open ponds. Arch. Hydrobiol. Beih. 20, 85-93.

22. Merchuk, J.C., Ronen, M., Giris, S., Arad, S. 1998. Light/dark cycles in the growth of the red microalga Porphyridium Sp. Biotech. Bioeng. 59, 705-713.

23. Miller, R.L., Tsuchiya, H.M., Fredrickson, A.G., Brown, A.H. 1964. Hydromechanical method to increase efficiency of algal photosynthesis. Ind. Eng. Chem. Proc. Dd. 3, 134-143.

24. Miron, A. S., Garcia, M.-C.C., Camacho, F.G., Grima, E.M., Chisti, Y. Growth and biochemical characterization of microalgal biomass produced in bubble column and airlift photobioreactors: studies in fed-batch culture. Enzyme and Microb. Tech. 31, 1015-1023.

25. Morweiser, M., Kruse, O., Hankamer, B., Posten. 2010. Developments and perspectives of photobioreactors for biofuel production. Appl. Microbio. Biotech. 87, 1291-1301.

26. Racina, A., Liu, Z., Kind, M. 2010. Mixing in Taylor-Couette flow, in: Bockhorn, H., Mewes, D., Peukert, W., Warnecke, H.-J. (Eds.), Micro and Macro Mixing: Analysis, Simulation and Numerical Calculation, Springer-Verlag, Berlin, pp. 125-139.

27. Ramezani, M., Kong, B., Gao, X., Olsen, M.G., Vigil, R.D. 2015. Experimental measurement of oxygen mass transfer and bubble size distribution in an air-water multiphase Taylor-Couette vortex bioreactor. Chem. Eng. J. 279, 286-296.

28. Sander, R. 2015. Compilation of Henry's law constants (version 4.0) for water as solvent. Atmos. Chem. and Phys. 15, 4399-4981. 
29. Sobczuk, T.M., Camacho, F.G., Grima, E.M., Chisti, Y. 2006. Effects of agitation on the microalgae Phaeodactylum tricornutum and Porphyridium cruentum. Bioproc. Biosys. Eng. 28, 243-250.

30. Spolaore, P., Joannis-Cassan, C., Duran, E., Isambert, A. 2006. Commercial applications of microalgae. J. Biosci. Bioeng. 101, 87-96.

31. Takache, H., Pruvost, J., Marec, H. 2015. Investigation of light/dark cycles effects on the photosynthetic growth of Chlamydomonas reinhardtii in conditions representative of photobioreactor cultivation. Algal Res. Biomass Biofuels Bioprod. 8, 192-204.

32. Tamimi, A., Rinker, E.B. Sandall, O.C. 1994. Diffusion coefficients for hydrogen sulfide, carbon dioxide, and nitrous-oxide in water over the temperature-range 293-368 K. J. Chem. and Eng. Data. 39, 330-332.

33. Thomas, W.H., Gibson, C.H. 1990. Effects of small-scale turbulence on microalgae. J. Appl. Phyc. 2, 71-77.

34. Ugwu, C.U., Ogbonna, J.C., Tanaka, H. 2005. Light/dark cyclic movement of algal culture (Synechocystis aquatilis) in outdoor inclined tubular photobioreactor equipped with static mixers for efficient production of biomass. Biotech. Lett. 27, 75-78. 


\section{Figure Captions}

Figure 1. Schematic diagram of the batch culture and data acquisition system.

Figure 2. Mixing time, $t_{m i x}$, as functions of (a) cylinder rotation speed and (b) gas flow rate. The same trends are observed independent of the choice of constant gas volumetric flow rate in (a) or constant cylinder speed in (b).

Figure 3. $\mathrm{CO}_{2}$ volumetric mass transfer coefficent, $k_{L} a$, as functions of (a) cylinder rotation speed and (b) gas flow rate. The same trends are observed independent of the choice of constant gas volumetric flow rate in (a) or constant cylinder speed in (b).

Figure 4. Experimental measurement of dissolved oxygen concentration in the Taylor vortex reactor as a percentage of water saturated with air at $20^{\circ} \mathrm{C}$. Inner cylinder rotation speed in both experiments was $400 \mathrm{rpm}$.

Figure 5. Dry biomass growth curves and yield plots showing the effect of gas composition (plots (a) and (b)), cylinder rotation speed (plots (c) and (d)), and gas flow rate (plots (e) and (f)).

Figure 6. Characteristic time scales for mixing $\left(t_{m i x}\right)$, mass transfer $\left(t_{M T}\right)$, and biomass growth $\left(t_{g}\right)$ plotted as a function of time for two typical C. vulgaris growth experiments. Two curves are shown for $t_{g}$, corresponding to calculations using Eqs. (15) and (18), respectively. 
Figure 1
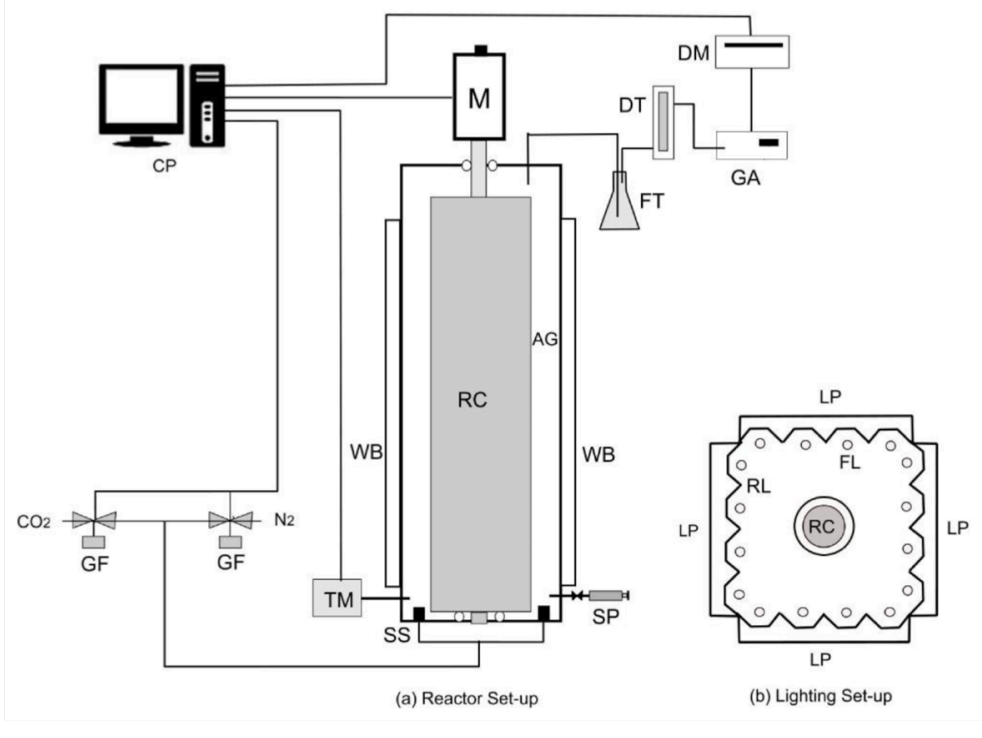

(b) 

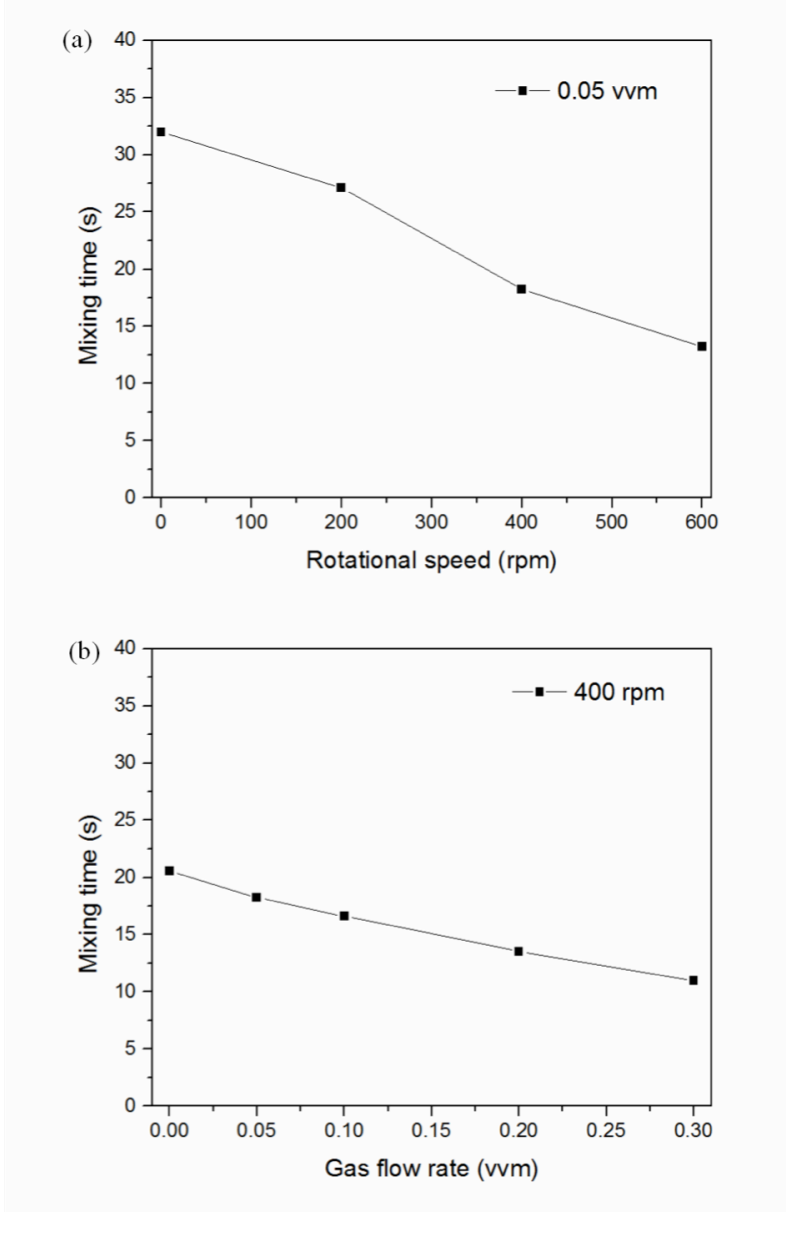

Figure 2 

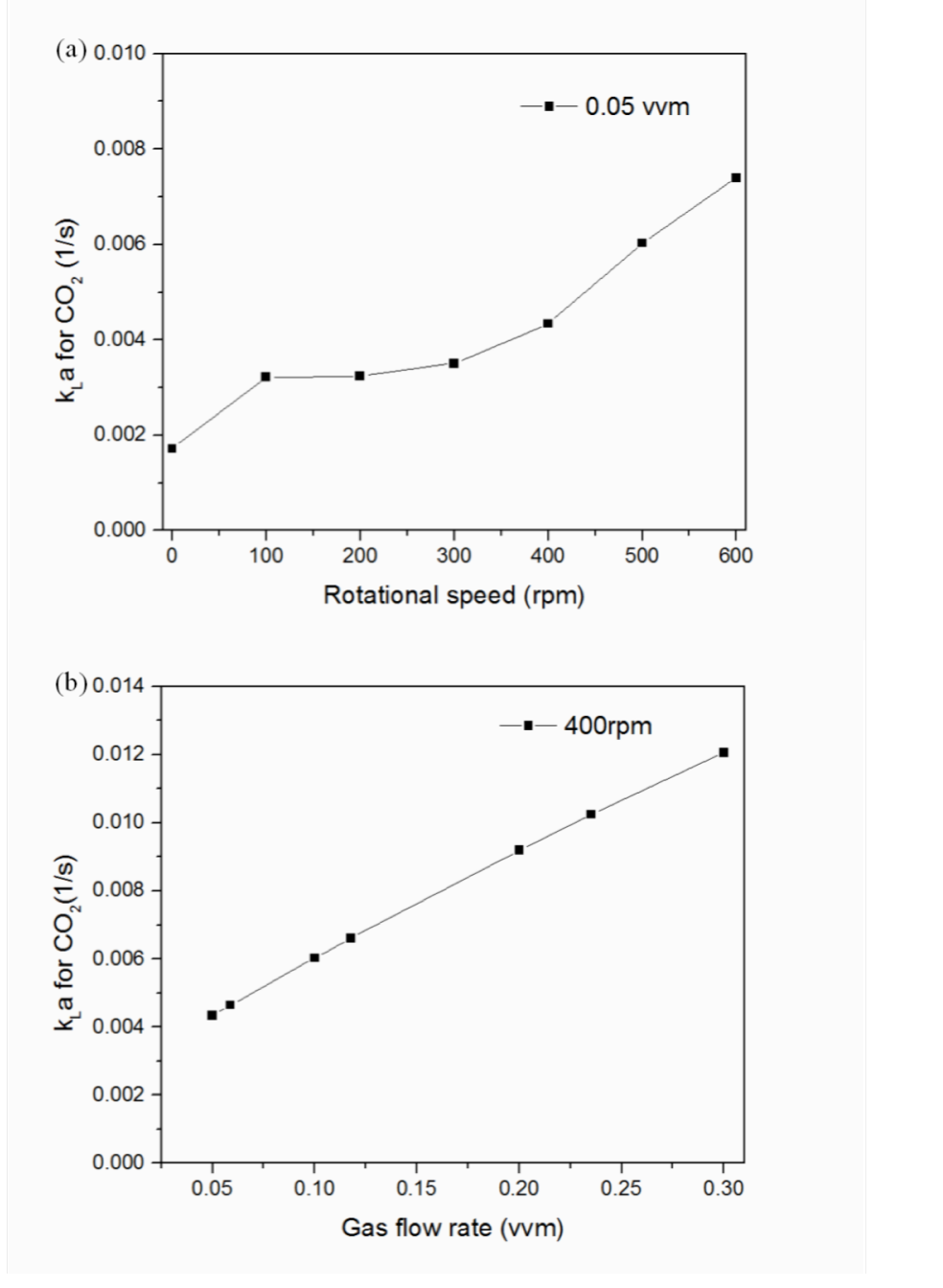

Figure 3

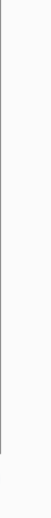

3

Gas flow rate (vvm)

.

.
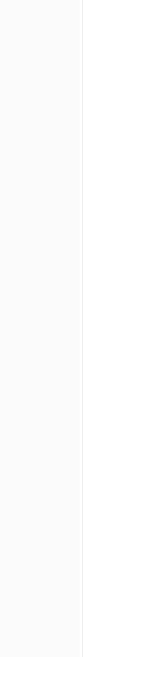

(a) 0.010
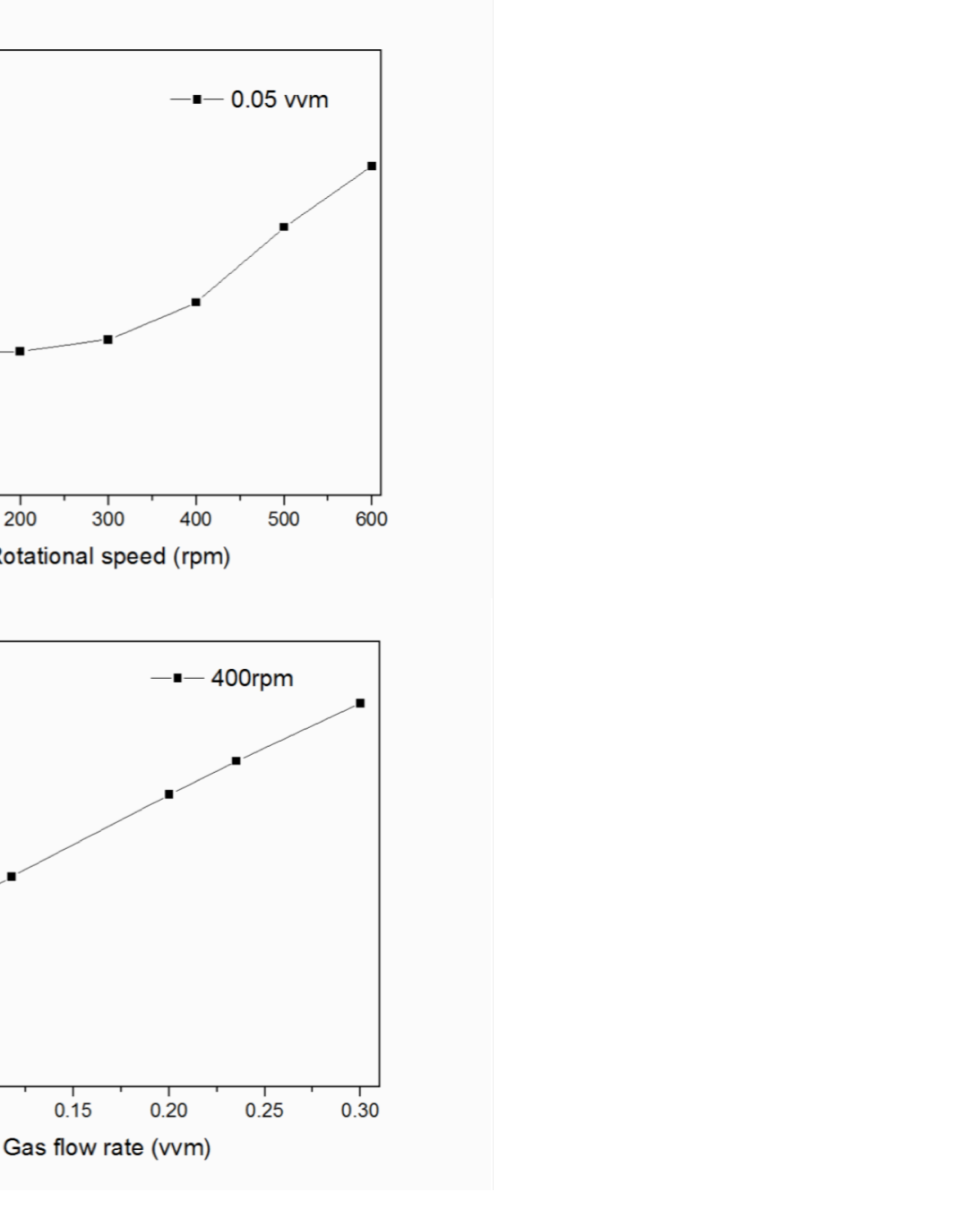
Figure 4
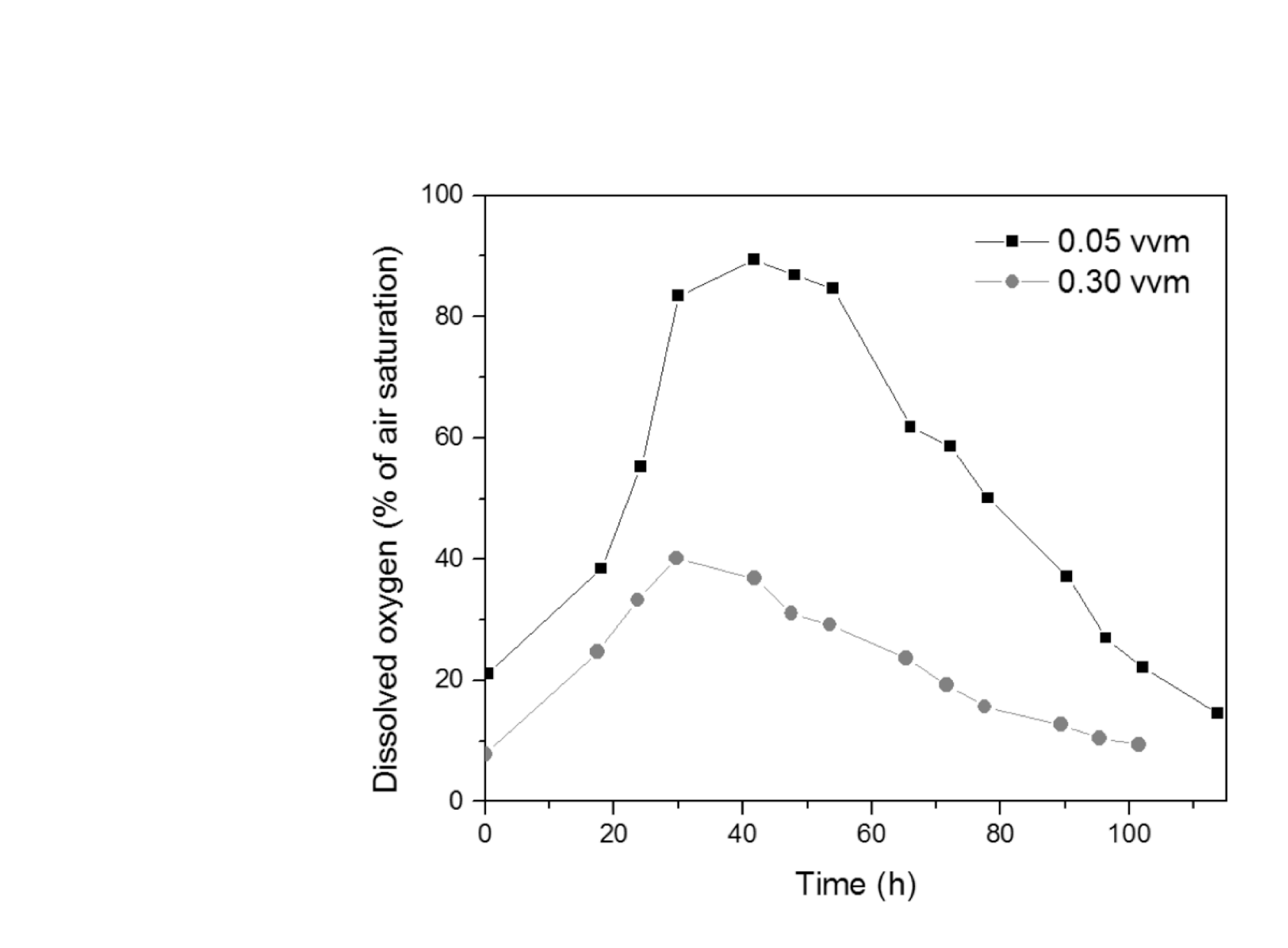

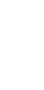


Figure 5
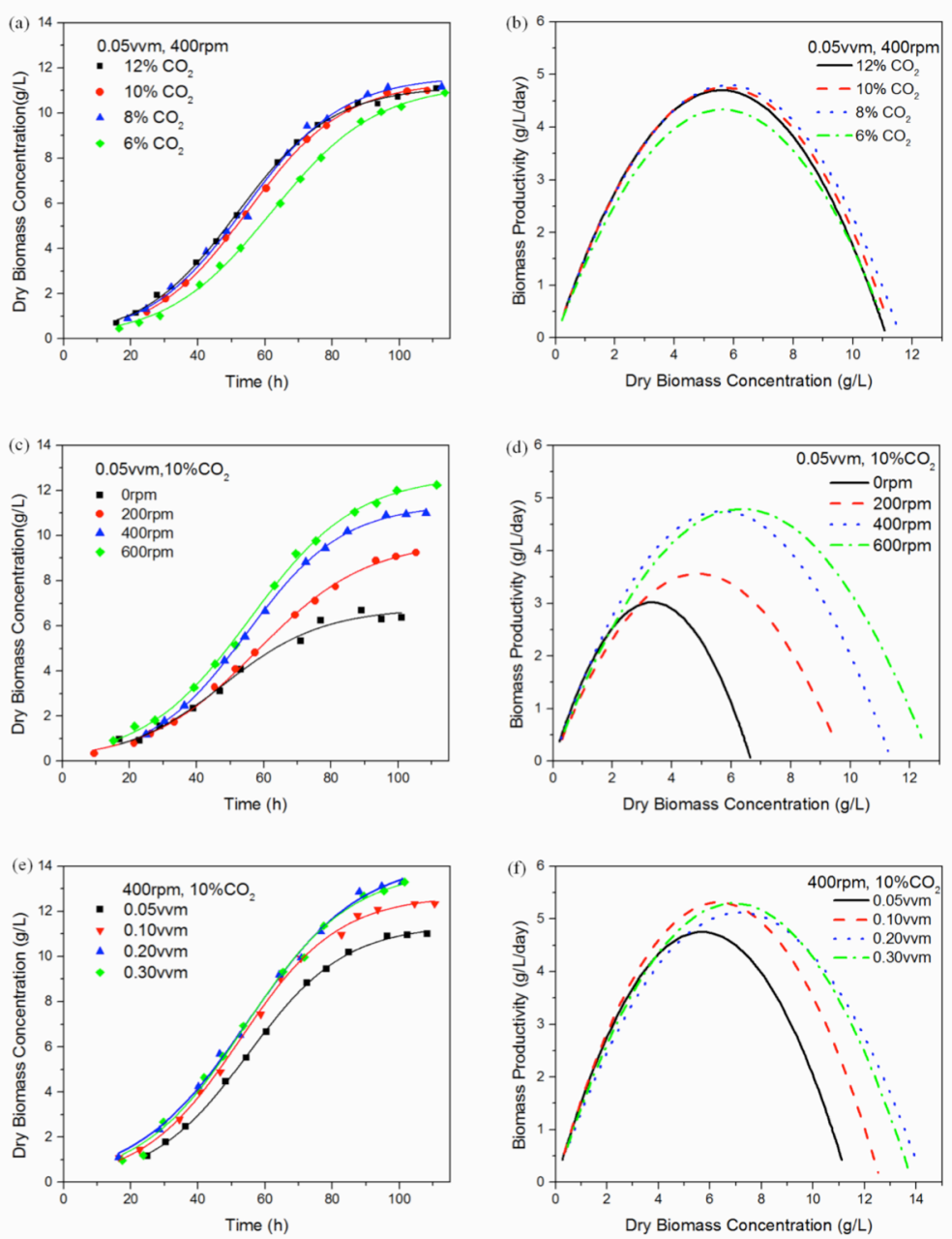

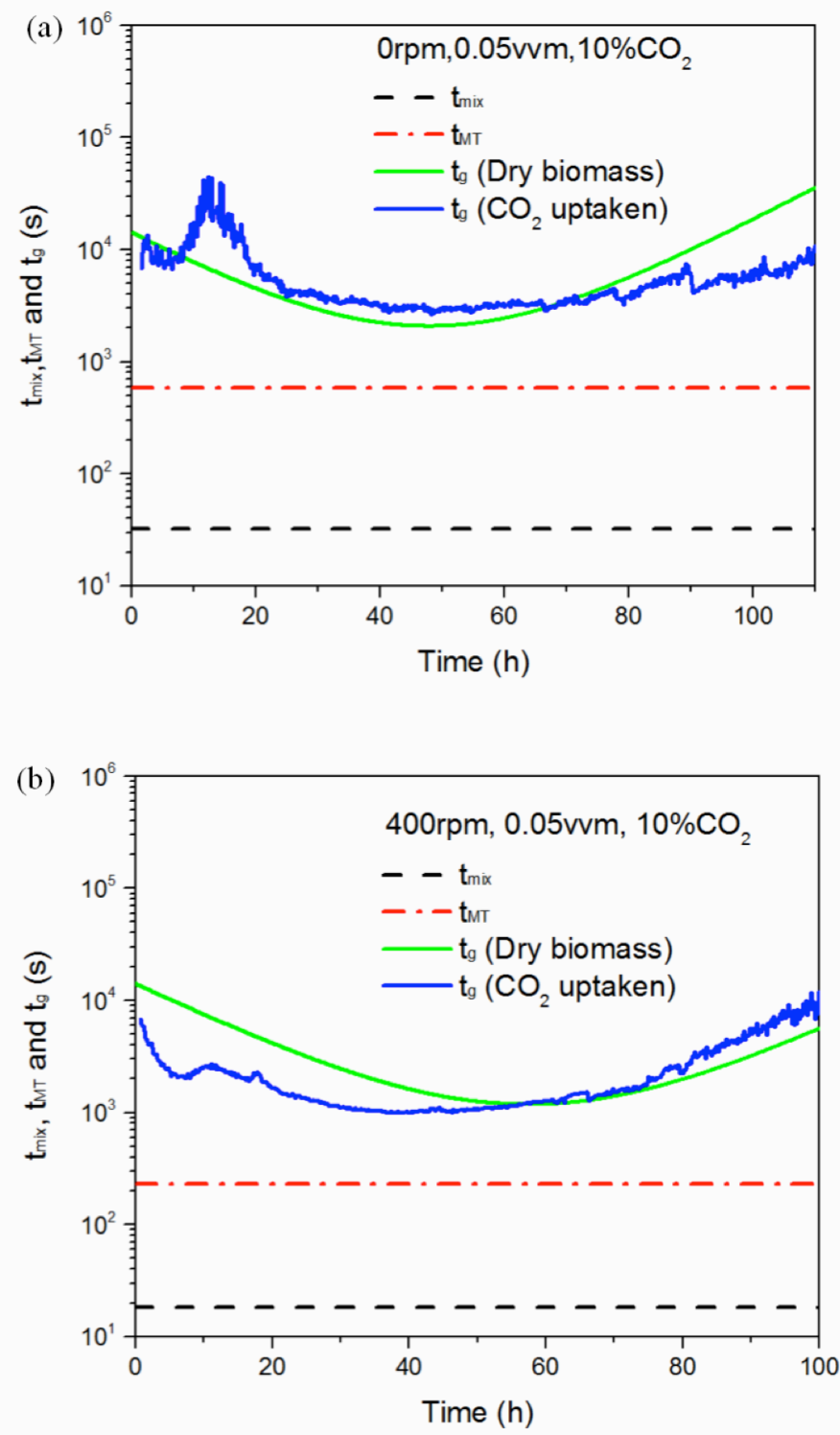